\title{
Immune checkpoint blockade therapies for HCC: current status and future implications
}

\author{
Ritu Shrestha', ${ }^{1,2}$ Kim R. Bridle ${ }^{1,2}$, Darrell H. G. Crawford ${ }^{1,2}$, Aparna Jayachandran ${ }^{1,2}$ \\ 'The University of Queensland, Faculty of Medicine, Brisbane, Queensland 4120, Australia. \\ ${ }^{2}$ Gallipoli Medical Research Institute, Greenslopes Private Hospital, Brisbane, Queensland 4120, Australia.
}

Correspondence to: Dr. Aparna Jayachandran, The University of Queensland, Faculty of Medicine, Lower Lobby Level, Administration Building, Greenslopes Private Hospital, Greenslopes, Queensland 4120, Australia.

E-mail: a.jayachandran@uq.edu.au

\begin{abstract}
How to cite this article: Shrestha R, Bridle KR, Crawford DHG, Jayachandran A. Immune checkpoint blockade therapies for HCC: current status and future implications. Hepatoma Res 2019;5:32. http://dx.doi.org/10.20517/2394-5079.2019.24
\end{abstract}

Received: 27 Jun 2019 First Decision: 11 Jul 2019 Revised: 6 Aug 2019 Accepted: 9 Aug 2019 Published: 3 Sep 2019

Science Editors: Jia Fan, Ying-Hong Shi Copy Editor: Jia-Jia Meng Production Editor: Jing Yu

\begin{abstract}
Hepatocellular carcinoma (HCC) is the most lethal and common type of liver cancer with limited treatment options at the advanced stage. The use of immune checkpoint inhibitor $(\mathrm{ICI})$ based immunotherapy is exponentially increasing in the treatment of patients with advanced solid tumors. The expression of immune checkpoints on tumor cells leading to lower activity of T-cells is one of the major mechanisms of immune escape. Checkpoint blockade immunotherapies with antibodies against PD-1, PD-L1 or CTLA-4 are being investigated in clinical trials in $\mathrm{HCC}$ patients. ICls have improved survival in patients with inoperable advanced stage $\mathrm{HCC}$ where other curative treatments are not applicable. However, the response rates remain low with only a small subset of patients responding to this therapy. There is an unmet need to identify predictive markers to select those HCC patients who would benefit from ICl therapies. Importantly, epithelial-to-mesenchymal transition (EMT), a major process driving HCC invasion and metastasis by regulating the phenotypic cellular switching from epithelial to mesenchymal state, has been implicated as a resistance mechanism associated with $\mathrm{ICl}$ therapies. The role of EMT as a regulator of immune checkpoint molecule in HCC is just emerging. However, the consequence of EMT as a resistance mechanism in HCC patients undergoing $\mathrm{ICl}$ treatments remains unexplored. In this review, we summarize the recent clinical studies with $\mathrm{ICls}$ in $\mathrm{HCC}$ and highlight the trials underway featuring novel monotherapies and combinatorial approaches based on immune and non-immune therapies. We will discuss the ongoing efforts to discover new immune checkpoint molecules in HCC as potential drug targets. We also highlight the role of EMT in facilitating therapy resistance in $\mathrm{HCC}$ treated with $\mathrm{ICl}$ and discuss potential strategies to circumvent resistance in $\mathrm{ICI}$ treated $\mathrm{HCC}$ patients.
\end{abstract}

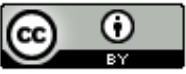

(C) The Author(s) 2019. Open Access This article is licensed under a Creative Commons Attribution 4.0 International License (https://creativecommons.org/licenses/by/4.0/), which permits unrestricted use, sharing, adaptation, distribution and reproduction in any medium or format, for any purpose, even commercially, as long as you give appropriate credit to the original author(s) and the source, provide a link to the Creative Commons license, and indicate if changes were made.

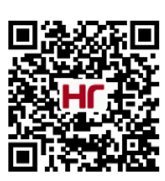


Keywords: Hepatocellular carcinoma, immunotherapy, immune checkpoint inhibitors, epithelial-to-mesenchymal transition, programmed cell death protein-1, programmed death-ligand 1, resistance

\section{INTRODUCTION}

Hepatocellular carcinoma (HCC) is the most frequent type of primary liver cancer and is associated with a high mortality rate ${ }^{[1]}$. The incidence of HCC is increasing annually by $3 \%-9 \%$ worldwide and the number of new cases and the number of deaths are almost in equal proportions ${ }^{[2]}$. Patients diagnosed with early stage HCC, have a better prognosis than advanced stage HCC patients with unresectable tumors ${ }^{[3]}$. Surgical resection and liver transplantation, the curative treatment approaches for early stage HCC provides 5-year survival rate of greater than $70 \%{ }^{[4,5]}$. Loco-regional therapies such as radiofrequency ablation (RFA), thermal and non-thermal ablation and transarterial chemoembolization (TACE) are also available as alternative treatment options for unresectable early stage $\mathrm{HCCs}^{[6-8]}$. However, the multi-targeted tyrosine kinase inhibitor (TKI) Sorafenib and Lenvatinib are the only first-line treatment available for the inoperable advanced stages of $\mathrm{HCC}^{[9]}$.

As the survival benefit with Sorafenib is limited to only 3 months ${ }^{[10]}$, several clinical trials have examined the suitability of new drugs for the treatment of patients with advanced stage HCC ${ }^{[11]}$. TKIs such as Regorafenib, Ramucirab, and Cabozanitib have been recently approved by the Food and Drug Administration (FDA) as second-line treatment alternatives for HCC patients previously treated with Sorafenib ${ }^{[12-15]}$. In addition, a combination therapy of TACE plus Sorafenib from the TCTICS trial also reported improved progression-free survival ${ }^{[1]}$. However, the limited survival benefit and associated toxicity with TKIs suggests an urgent need for better and efficacious treatment approaches for advanced stage HCC.

Immunotherapy has emerged as a potential alternative in the treatment of cancers following the clinical success of immune checkpoint inhibitors (ICIs). ICIs target the negative immune regulatory pathways such as cytotoxic T lymphocyte-associated protein 4 (CTLA-4) and the programmed cell death protein-1/ programmed cell death ligand 1 (PD-1/PD-L1) which inhibit T-cell immune response. ICI treatments have demonstrated dramatic anti-tumor clinical effects in several malignancies including melanoma, lung cancer and renal cell carcinoma ${ }^{[16-19]}$. Immunotherapeutic approaches based on ICIs have substantially enhanced disease-free survival in HCC patients resulting in the approval of anti PD-1 monoclonal antibodies, Nivolumab and Pembrolizumab, as second-line treatment options for advanced HCC $^{[20-22]}$. Notably, Nivolumab increases survival in HCC patients to 17 months, far exceeding the 3 months extension in survival offered by Sorafenib ${ }^{[20]}$.

In this review, we will highlight the clinical trials that address the utility of ICIs as therapeutic tools in the management of HCC. We will focus on ICIs as monotherapies and combination therapy regimen for HCC patients. Although ICIs have proven to be effective, therapeutic resistance occurs in the majority of patients, leading to tumor progression. We explore EMT process as a main resistance mechanism to immune checkpoint blockade therapy and review studies that link EMT to immune checkpoint regulation.

\section{IMMUNOTHERAPY BASED ON IMMUNE CHECKPOINT BLOCKADE}

Immune equilibrium is vital for preventing uncontrolled immune responses leading to severe inflammatory conditions or autoimmune disorders ${ }^{[23,24]}$. The immune equilibrium is maintained by balance between coinhibitory and co-stimulatory signals that regulate T-cell activation ${ }^{[23-25]}$. T-cells are activated when specific antigens are recognized by T-cell receptors, whereas, the immune checkpoints provide an inhibitory effect on the activation of T-cells ${ }^{[23,24]}$. Immune checkpoint molecules are thus responsible for self-tolerance and 
prevent immune overstimulation in normal conditions ${ }^{[23,24]}$. However, the cancer cells hijack these immune checkpoint molecules to bypass T-cell-mediated cytotoxicity resulting in tumor immune evasion ${ }^{[26]}$.

ICIs are the class of immunotherapeutic drugs including monoclonal antibodies against immune checkpoint molecules that stops the inhibitory effects of immune checkpoint molecules on T-cells resulting in the restoration of immune-mediated antitumor activity ${ }^{[16,27]}$. The first ICI drug approved by FDA for cancer immunotherapy was Ipilimumab (anti-CTLA-4) for treatment of advanced melanoma ${ }^{[26]}$. The PD-1/PD-L1 pathway along with CTLA-4 are the most studied and targeted molecules in cancer immunotherapeutic research and clinical trials ${ }^{[28]}$. Several other immune checkpoint molecules have also been assessed as potential targets such as TIM-3, BTLA, VISTA, LAG-3, VTCN1, CD73, B7-H3 and OX $40^{[23,25]}$. ICIs have shown clinical benefits in several other cancers such as lung cancer and renal cell carcinoma following its approval in melanoma ${ }^{[16-19]}$.

\section{FEASIBILITY OF IMMUNE CHECKPOINT BLOCKADE IN HCC}

Immune checkpoint blockade therapy can be exploited as an alternative treatment approach in HCC similar to other cancers, as liver possess a unique immunobiology ${ }^{[2]]}$. The tumor microenvironment (TME) in HCC is known to play a vital role in immune activation or suppression contributing to either tumor eradication or tumor progression ${ }^{[6,30]}$. The strong intrinsic immune suppressive microenvironment of the liver results in intrahepatic tolerogenicity ${ }^{[6,31]}$. Some of the key players contributing to immunological tolerance in liver are liver sinusoidal endothelial cells, Kupffer cells and hepatic dendritic cells ${ }^{[6]}$. This immune suppressive microenvironment is more evident during formation and progression of HCC depending on several mechanisms including expression of immune checkpoint molecules leading to the development of an anti-tumor immunity ${ }^{[6,32]}$. These immune evasive abilities of HCC make immunotherapy a plausible therapeutic option in HCC. Several clinical studies have already reported efficacy of ICI drugs in HCC. However, only two ICI drugs, Nivolumab and Pembrolizumab, have been approved for HCC patients previously treated with Sorafenib based on the CheckMate 040 trial and Keynote-224 trial respectively ${ }^{[20,22]}$.

\section{ICIS IN THE CLINICAL MANAGEMENT OF HCC}

Several clinical trials have been conducted and many others are ongoing in HCC including ICIs alone or in combination with other therapeutic agents. The clinical studies of ICIs in HCC constitute targeting PD-1, PD-L1 and CTLA-4. The key findings from some major earlier clinical studies of ICIs in HCC are summarized in Table 1. The clinical immune checkpoint blockade studies have either been as a monotherapy or combination therapy.

\section{ICI AS MONOTHERAPY IN HCC}

ICIs have been used as monotherapy in several clinical studies for HCC as summarized in Table 2.

\section{ICIS BLOCKING CTLA-4}

CTLA-4 is a protein receptor expressed on activated T-cells and Tregs which binds to CD80 and CD86 upon stimulation such that it blocks the binding of CD28 to CD80 and CD86 and inhibits T-cell activation $^{[23,33]}$. A study has shown that treatment with anti-CTLA-4 antibody resulted in increased frequency of tumor-associated antigens such as interleukin (IL)-1, IL-6 and macrophage inflammatory protein-1 in $60 \%$ of HCC patients ${ }^{[34]}$.

\section{Tremelimumab}

In HCC, the first clinical trial using ICI was Tremelimumab, anti-CTLA-4, reported by Sangro et al. ${ }^{[35]}$. In this trial, HCC patients with chronic Hepatitis C viral infection were treated with Tremelimumab 
Table 1. Findings of initial clinical studies of immune checkpoint inhibitors in hepatocellular carcinoma

\begin{tabular}{|c|c|c|c|c|c|c|}
\hline Target & Immune checkpoint inhibitor & Phase & Overall survival & Clinical trial number & Approval & Reference \\
\hline \multirow[t]{2}{*}{ PD-1 } & Nivolumab & $\mathrm{I} / \mathrm{II}$ & 15 months dose escalation & NCT01658878 & Approved & {$[20]$} \\
\hline & Pembrolizumab & II & 12.9 months & NCT02702414 & Approved & {$[22]$} \\
\hline CTLA-4 & Tremelimumab & II & 8.2 months & NCT01008358 & Not approved & [35] \\
\hline PD-L1 & Durvalumab & I/II & 13.2 months & NCT01693562 & Not approved & {$[51]$} \\
\hline PD-L1 and CTLA-4 & Durvalumab + Tremelimumab & $\mathrm{I} / \mathrm{II}$ & Not reported & NCT02519348 & Not approved & {$[55]$} \\
\hline CTLA-4 and ablation & Tremelimumab + ablation & & 12.3 months & NCT01853618 & Not approved & {$[64]$} \\
\hline
\end{tabular}

PD-1: programmed death protein-1; CTLA-4: cytotoxic T lymphocyte-associated protein-4; PD-L1: programmed death protein ligand -1

Table 2. Current clinical trials of immune checkpoint inhibitors as monotherapy in hepatocellular carcinoma

\begin{tabular}{lllllll}
\hline Target & Immune checkpoint inhibitor & Phase & Clinical trial number & \multicolumn{1}{c}{ Design } & Lines of therapy & End point \\
\hline PD-1 & Nivolumab & III & NCT02576509 & Nivolumab vs. Sorafenib & First-line therapy & OS \\
& Nivolumab & III & NCT03383458 & Nivolumab vs. placebo & Adjuvant therapy & PFS \\
& Pembrolizumab & III & NCT03062358 & Pembrolizumab vs. placebo & Second-line therapy & OS \\
& Pembrolizumab & II & NCT03337841 & Pembrolizumab & Neoadjuvant therapy & RFS \\
& Tislelizumab & II & NCT03419897 & Tislelizumab & Second-line therapy & ORR \\
& Tislelizumab & III & NCT03412773 & Tislelizumab vs. Sorafenib & First-line therapy & OS \\
& Camrelizumab & II/III & NCT02989922 & Camrelizumab & Second-line therapy & ORR/OS \\
& Avelumab & II & NCT03389126 & Avelumab & Second-line therapy & ORR \\
\hline
\end{tabular}

PD-1: programmed death protein-1; PD-L1: programmed death protein ligand -1; OS: overall survival; PFS: progression free survival; RFS: recurrence free survival; ORR: overall response rate

and 3 out of 17 assessable patients showed partial responses (17.6\%) and an additional 10 patients (58.8\%) had stable disease resulting in time-to-progression of 6.48 months and overall survival of 8.2 months $(\mathrm{NCT} 01008358)^{[35,36]}$. Tremelimumab is the only anti-CTLA-4 ICI which is undergoing a phase III trial as monotherapy in HCC as of September $2018^{[28]}$.

\section{ICIS BLOCKING PD-1}

$\mathrm{PD}-1$, a key regulator of T-cell mediated immune response, is expressed by activated T cells, B-cells, natural killer cells, Tregs, myeloid-derived suppressor cells (MDSCs), monocytes and dendritic cells ${ }^{[37]}$.

\section{Nivolumab}

Nivolumab is the first recombinant monoclonal human IgG4 antibody specific for PD-1 ${ }^{[11]}$. Nivolumab is also the first FDA approved ICI for HCC based on the CheckMate 040 trial (NCT01658878) ${ }^{[20]}$. The phase I/II study of CheckMate 040 trial with 262 treated patients and 202 patients with complete treatment reported a response rate of $20 \%$ with three complete responses and 39 partial responses in patients with advanced HCC and Child-Pugh A cirrhosis who progressed on or were intolerant to Sorafenib ${ }^{[20]}$. There are several ongoing clinical trials for Nivolumab in HCC either as monotherapy or in combination. The success of earlier clinical studies of Nivolumab led to a phase III clinical trial CheckMate 459 (NCT02576509) examining Nivolumab as a first-line therapy in HCC and comparing the effects with Sorafenib in 726 HCC patient ${ }^{[38]}$. However, a press release from Bristol-Myers Squibb recently announced that the topline results from the phase III clinical trial CheckMate 459 failed to meet its primary endpoint of overall survival. Nivolumab is also being studied as an adjuvant therapy after surgical resection or ablation therapy in a second phase III trial CheckMate 9Dx (NCT03383458) ${ }^{[28]}$. There are several ongoing clinical trials for Nivolumab in HCC either as monotherapy or in combination with other therapies.

\section{Pembrolizumab}

Pembrolizumab is another recombinant monoclonal human IgG4 antibody specific for human PD-1. Pembrolizumab gained approval for HCC patients previously treated with Sorafenib in November 2018 
based on a phase II clinical study of HCC patients, Keynote-224 (NCT02702414) that reported an overall response rate of $17 \%$ among 104 patients with 1 complete response and 16 partial responses ${ }^{[22]}$. A clinical study with 450 Asian HCC patients to evaluate efficacy and safety of Pembrolizumab or placebo with best supportive care (NCT03062358) is ongoing ${ }^{[16]}$. Another study is examining Pembrolizumab before and after surgery or ablation to evaluate HCC recurrence (NCT03337841) ${ }^{[16]}$. Recently, a phase III clinical study Keynote-240 investigating Pembrolizumab plus best supportive care compared to placebo plus best supportive care failed to meet its co-primary endpoints of overall survival and progression free survival in 413 patients with advanced HCC previously treated with systemic therapy ${ }^{[39]}$. Similar to Nivolumab, there are several ongoing trials of Pembrolizumab in HCC either as monotherapy or in combination with other treatments.

\section{Tislelizumab}

Tislelizumab is also another human IgG4 against PD- ${ }^{[40]}$. A phase I trial of Tislelizumab in 61 patients with solid cancers including HCC confirmed the safety of this drug ${ }^{[2]}$. In HCC, Tislelizumab is undergoing two clinical studies, one is a phase II clinical study assessing safety, efficacy and pharmacokinetics of the drug in 228 previously treated unresectable HCC patients (NCT03419897) and another is a phase III clinical study that compares safety and efficacy of Tislelizumab with Sorafenib as first line systemic treatment in 660 patients with unresectable HCC (NCT03412773) ${ }^{[28,41]}$.

\section{Camrelizumab}

Camrelizumab is a human IgG4 mAb against PD-1 which was reported to exhibit an anti-tumor response in 58 patients with solid cancers including HCC in a phase I trial ${ }^{[42,43]}$. Currently, several clinical studies are ongoing with Camrelizumab in HCC either alone or in combination with other treatments ${ }^{[40]}$. A phase II/III trial of Camrelibzumab reported a response rate of $13.8 \%$ and 6 month overall survival rate of $74.7 \%$ in HCC patients previously treated with systemic treatment $(\mathrm{NCT} 02989922)^{[4]]}$.

\section{ICIS BLOCKING PD-L1}

$\mathrm{PD}-\mathrm{L} 1$ is the main ligand for PD-1 that is responsible for suppression of T-cell migration, proliferation and secretion of cytotoxic mediators ${ }^{[45,46]}$. Studies have shown that higher expression of PD-L1 is associated with poor prognosis in HCC patients ${ }^{[25,47-50]}$. A study reported that PD-L1 expression by neoplastic and intratumoral inflammatory cells was associated with tumor aggressiveness ${ }^{[47]}$.

\section{Durvalumab}

Durvalumab is an anti-PD-L1 antibody which has been approved for treatment of advanced or metastatic urothelial carcinoma and non-small cell lung cancer ${ }^{[40]}$. Durvalumab was reported with a $10 \%$ response rate and median survival of 13.2 months in a cohort of 40 HCC patients in a phase I/II clinical study of Durvalumab monotherapy for solid cancers including HCC (NCT01693562) ${ }^{[51]}$.

\section{Avelumab}

Avelumab is a human IgG1 mAb targeting PD-L1 with ongoing trials for both monotherapy and combination therapy in $\mathrm{HCC}^{[40]}$. A phase II study of Avelumab is ongoing with $30 \mathrm{HCC}$ patients previously treated with Sorafenib (NCT03389126) ${ }^{[40]}$.

\section{ICI AS COMBINATION THERAPY IN HCC}

Despite promising results from clinical studies of ICIs as monotherapy in HCC, only a small patient population benefit from specific immune checkpoint blockade therapy ${ }^{[52]}$. Thus, several combination approaches have been utilized to improve the efficacy of ICI therapy. In HCC, the combination of anti-CTLA-4 and anti- PD-1/PD-L1 along with combinations of ICIs with other immune and non- 
Table 3. Current clinical trials of immune checkpoint inhibitors as combination therapy in hepatocellular carcinoma

\begin{tabular}{|c|c|c|c|c|}
\hline Target & Study design & Clinical trial number & Phase & End point \\
\hline \multicolumn{5}{|c|}{ Combination with other immune-based therapies } \\
\hline \multirow[t]{6}{*}{ PD-1 and CTLA-4 } & Nivolumab + Ipilimumab & NCT03682276 & $\mathrm{I} / \mathrm{II}$ & ORR \\
\hline & Nivolumab + Ipilimumab & NCT03510871 & $\|$ & \\
\hline & Nivolumab +/- Ipilimumab & NCT03222076 & II & Safety \\
\hline & Nivolumab +/- Ipilimumab & NCT03203304 & I & Safety \\
\hline & $\begin{array}{l}\text { Tremelimumab vs. Tremelimumab + Durvalumab vs. } \\
\text { Sorafenib }\end{array}$ & NCT03298451 & III & OS \\
\hline & $\begin{array}{l}\text { Tremelimumab vs. Durvalumab vs. Tremelimumab + } \\
\text { Durvalumab }\end{array}$ & NCT02519348 & II & Safety \\
\hline PD-L1 and TIM-3 & LY3300054 +/- LY3321367 & NCT03099109 & I & Safety \\
\hline PD-1 and LAG-3 & REGN2810 +/- REGN3767 & NCT03005782 & I & Safety/ORR \\
\hline \multicolumn{5}{|c|}{ Combination with molecular targeted agents } \\
\hline PD-L1 and anti-VEGF & Atezolizumab + Bevacizumab & NCT02715531 & I & Safety/ORR \\
\hline PD-L1 and anti-VEGF & Atezolizumab + Bevacizumab vs. Sorafenib & NCT03434379 & III & OS/ORR \\
\hline PD-1 and TKI & Pembrolizumab + Lenvatinib vs. Lenvatinib & NCT03713593 & III & PFS/OS \\
\hline PD-1 and TKI & Pembrolizumab + Lenvatinib & NCT03006926 & I & Safety/OR/DOR \\
\hline PD-1 and TKI & Camrelizumab (SHR-1210) + Apatinib & NCT02942329 & $\mathrm{I} / \mathrm{II}$ & OS \\
\hline PD-1 and TKI & Spartalizumab (PDR001) + Sorafenib & NCT02988440 & I & Safety \\
\hline $\begin{array}{l}\text { PD-1 and c-MET } \\
\text { inhibitor }\end{array}$ & Spartalizumab (PDR001) +/- Capmatinib (INC280) & NCT02795429 & I/II & Safety/ORR \\
\hline PD- 1 and anti-TGF- $\beta$ & Spartalizumab (PDR001) +/- NIS793 & NCT02947165 & I & Safety \\
\hline $\begin{array}{l}\text { PD-1 and FGFR4 } \\
\text { inhibitor }\end{array}$ & Spartalizumab (PDR001) +/- FGF401 & NCT02325739 & $1 / I I$ & Safety/TTP/ORR \\
\hline PD-1 and TKI & Nivolumab +/- Lenvatinib & NCT03418922 & I & Safety \\
\hline PD-1 and TKI & Nivolumab + Cabozatinib & NCT03299946 & I & $\begin{array}{l}\text { Safety/ } \\
\text { Completion }\end{array}$ \\
\hline PD-1 and anti-VEGF & Nivolumab + Bevacizumab & NCT03382886 & । & Safety \\
\hline PD-1 and TKI & Pembrolizumab + Regorafenib & NCT03347292 & । & Safety \\
\hline PD-1 and TKI & Pembrolizumab + Sorafenib & NCT03211416 & $I / I I$ & ORR \\
\hline PD-L1 and TKI & Avelumab + Axitinib & NCT03289533 & । & Safety \\
\hline $\begin{array}{l}\text { PD-L1 and DNMT } \\
\text { inhibitor }\end{array}$ & Durvalumab + Guadecitabine & NCT03257761 & I & Safety/ORR \\
\hline $\begin{array}{l}\text { CTLA-4, PD-1 and } \\
\text { anti-OX40 }\end{array}$ & $\begin{array}{l}\text { Nivolumab + INCAGN01949 vs. Ipilimumab + } \\
\text { INCAGN01949 vs. Nivolumab + Ipilimumab + } \\
\text { INCAGN01949 }\end{array}$ & NCT03241173 & $\mathrm{I} / \mathrm{II}$ & Safety/ORR \\
\hline $\begin{array}{l}\text { PD-1 and anti- } \\
\text { phosphatidyl-serine }\end{array}$ & Pembrolizumab + Bavituximab & NCT03519997 & II & ORR \\
\hline \multicolumn{5}{|c|}{ Combination with local therapies } \\
\hline PD-1 and ischemia & Nivolumab + TACE & NCT03143270 & I & Safety \\
\hline PD-1 and radiation & Pembrolizumab + TACE & NCT03397654 & $I / I I$ & Safety \\
\hline PD-1 and radiation & Nivolumab + Y90 & NCT03033446 & ॥ & ORR \\
\hline $\begin{array}{l}\text { CTLA-4, PD-L1 and } \\
\text { ischemia }\end{array}$ & Tremelimumab + Durvalumab + Radiation & NCT03482102 & ॥ & ORR \\
\hline $\begin{array}{l}\text { PD- } 1 \text { and HSV } \\
\text { oncolytic virus }\end{array}$ & Pembrolizumab +/-Talimogene Laherparepvec (T-VEC) & NCT2509507 & I & Safety/ORR \\
\hline
\end{tabular}

PD-1: programmed death protein-1; CTLA-4: cytotoxic T lymphocyte-associated protein-4; PD-L1: programmed death protein ligand -1; TKI: tyrosine kinase inhibitor, OS: overall survival; PFS: progression free survival; RFS: recurrence free survival; ORR: overall response rate; TTP: time to progression

immune based treatment approaches are being studied. The combination therapies with ICI for HCC are summarized in Table 3.

\section{IMMUNE-BASED COMBINATION THERAPIES FOR HCC}

The blockade of CTLA-4 and PD-1/PD-L1 is the most promising ICI combination therapy that could enhance the anti-tumor effects in HCC. This combination blockade therapy has been very effective as an immune dampener as CTLA-4 signaling prevents the initiation of a T-cell response, while the PD-1/PD-L1 axis limits T-cell activity in the $\mathrm{TME}^{[28]}$. 


\section{Ipilimumab (anti-CTLA4) + Nivolumab (anti-PD-1)}

Since its FDA approval in 2011 for advanced melanoma, Ipilimumab (anti-CTLA4) has also been approved for renal cell carcinoma in combination with another ICI, Nivolumab (anti-PD-1), based on CheckMate $214^{[53,54]}$. In HCC, there are four ongoing trials combining Ipilimumab with other $\mathrm{ICII}^{[40]}$. The first study is the combination therapy of Ipilimumab and Nivolumab for HCC patients before liver resection $(\mathrm{NCT} 03682276)^{[40]}$. The second study is also a combination therapy with Nivolumab as neoadjuvant therapy for HCC (NCT03510871 ${ }^{[40]}$. A third study compares the combination of Ipilimumab and Nivolumab versus Nivolumab alone in resectable HCC (NCT03222076) ${ }^{[40]}$. The fourth study also compares combination of Ipilimumab and Nivolumab with Nivolumab alone in terms of safety and tolerability, after external beam photon stereotactic body radiotherapy in patients with unresectable HCC (NCT03203304) ${ }^{[40]}$.

\section{Tremelimumab (anti-CTLA4) + Durvalumab (anti-PD-L1)}

A phase I/II clinical study including combination of Tremelimumab (anti-CTLA4) and Durvalumab (anti-PD-L1) in 40 HCC patients reported a response rate of $25 \%$ and manageable toxicity profile ${ }^{[55]}$. Currently, a phase III study of combination therapy including various dosage regimens of Durvalumab and Tremelimumab versus Sorafenib is ongoing to compare the efficacy of these therapeutic approaches $\left(\right.$ NCT03298451) ${ }^{[56]}$. Similar combination therapy of Tremelimumab and Durvalumab is being studied in a phase II trial in HCC patients previously treated with Sorafenib (NCTo2519348) ${ }^{[52]}$.

\section{Other ICl combinations}

Besides CTLA-4, other immune checkpoint molecules such as TIM-3 and LAG-3 are also being examined in combination with PD-1/PD-L1 blockade therapy ${ }^{[52]}$. There are ongoing clinical studies with combination of anti-TIM3 antibody LY3321367 with anti-PD-L1 antibody LY3300054 (NCT03099109), anti-LAG-3 antibody REGN3767 with or without the anti-PD-1 antibody REGN2810 (NCT03005782) ${ }^{[16]}$.

\section{NON-IMMUNE-BASED COMBINATION TREATMENTS WITH ICIS}

The effects of ICI therapy in HCC could be enhanced when combined with non-immune-based therapies such as chemotherapy with the aim to improve anti-tumor efficacy and survival in HCC.

\section{Atezolizumab (anti-PD-L1) + Bevacizumab (anti-VEGF)}

Atezolizumab is a human IgG1 mAb against PD-L1 which is being studied in combination with Bevacizumab (anti-VEGF antibody) in several clinical studies ${ }^{[57,58]}$. A phase I study of Atezolizumab and Bevacizumab as combination therapy reported a tolerable safety profile and promising response rates in patients (NCT02715531) ${ }^{[58]}$. Another phase III trial is ongoing for combination of Atezolizumab and Bevacizumab with 480 patients with advanced or metastatic HCC (NCT03434379 $)^{[57]}$.

\section{Pembrolizumab (anti-PD-1) + Lenvatinb (multikinase inhibitor)}

Pembrolizumab (anti-PD-1) in combination with Lenvatinib (a multikinase inhibitor) is currently being compared with Lenvatinib plus placebo as first-line treatment option in 750 HCC patients (NCT03713593) ${ }^{[59]}$. The combination therapy of Pembrolizumab and Lenvatinib reported a $42 \%$ response rate and median progression free survival of 9.69 months in HCC patients as per results presented at the ASCO $2018^{[28,57]}$. Another study is also ongoing with combination therapy of Pembrolizumab and Lenvatinib $(\mathrm{NCT} 03006926)^{[16]}$.

\section{Camrelizumab (anti-PD-1) + Apatinib (TKI)}

Camrelizumab (SHR-1210) is an anti-PD-1 antibody, which in combination with Apatinib, a TKI, has been reported at the ASCO 2018 meeting in a phase I trial with 18 HCC patients to demonstrate a response rate of $38.9 \%$ and a median progression free survival of 7.2 months (NCT02942329) ${ }^{[60]}$. 


\section{Spartalizumab (anti-PD-1) + other agents}

Spartalizumab is a human IgG4 mAb against PD-1 that is currently being studied in combination with other drugs such as Sorafenib (NCT02988440), Capmatinib (c-Met inihibitor) (NCT02795429), NIS793 (anti-TGF- $\beta$ ) (NCT02947165) and FGF401 (fibroblast growth factor receptor 4 inhibitor) (NCT02325739) ${ }^{[40]}$.

\section{Other combinations}

Several studies are ongoing for other combination of ICIs with molecular targeted agents such as Nivolumab + Lenvatinib (NCT03418922), Nivolumab + Cabozantinib (NCT03299946), Nivolumab + Bevacizumab (NCT03382886), Pembrolizumab + Regorafenib (NCT03347292), Pembrolizumab + Sorafenib (NCT03211416), Avelumab + Axitinib (NCT03289533), and others ${ }^{[28]}$.

There are ongoing early-phase studies with a combination of ICIs with other therapeutic agents such as the DNA methyltransferase (DNMT) inhibitor Guadecitabine (NCT03257761), the anti-OX40 mAb INCAGN01949 (NCT03241173), the anti-phosphatidylserine mAb Bavituximab (NCT03519997) and others $^{[16]}$.

\section{COMBINATION WITH LOCAL THERAPY}

Strategies to improve the potential efficacy of ICIs in HCC are being investigated in several ongoing clinical trials by including the addition of other conventional therapies such as TACE, RFA and other local therapies. Radiotherapy has been demonstrated to provide synergistic effect in combination with PD-L1 or CTLA-4 inhibitors ${ }^{[61,62]}$.

\section{Nivolumab (anti-PD-1) + local therapy}

Nivolumab in combination with TACE using drug-eluting beads is under study to assess the safety of this combination in a phase I trial (NCT03143270) ${ }^{[63]}$.

\section{Pembrolizumab (anti-PD-1) + TACE}

A phase I/II study of Pembrolizumab post TACE is evaluating safety and efficacy of the combination therapy $(\mathrm{NCT} 03397654)^{[28]}$.

\section{Tremelimumab (anti-CTLA4) + RFA or TACE}

Tremelimumab was also examined in combination therapy with RFA or TACE to test if tumor necrosis could induce antigenic stimulation and systemic immune response enhanced by immune checkpoint blockade (NCT01853618) ${ }^{[16,64]}$. This study resulted in partial response in 5 patients (26\%) out of 19 evaluable patients and 12 patients (63\%) had stable disease with time to progression 7.4 months and median overall survival of 12.3 months ${ }^{[16,64]}$.

\section{Other combinations with local therapies}

In addition to above mentioned trials, there are several other clinical studies ongoing to assess the combination of ICIs with local therapies including Nivolumab plus radioembolisation using yttrium-90 (NCT03033446), Durvalumab + Tremelimumab combined with radiotherapy (NCT03482102), Pembrolizumab with the oncolytic viral preparation Talimogene Laherparepvec (NCT02509507) and others ${ }^{[28,65,66]}$. These studies suggest another therapeutic option for treating chemoresistant cancer may become available.

\section{OVERCOMING THE LIMITATIONS OF IMMUNE CHECKPOINT BLOCKADE THERAPY}

Despite the clinical success with immune checkpoint blockade therapy, there have been several limitations. One of the major limitations of using ICIs is the associated significant adverse events from 
the therapy ${ }^{[28,52,67]}$. Johnson et al ${ }^{[68]}$ have reported two cases of lethal myocarditis in melanoma patients treated with combination of Nivolumab and Ipilimumab. In HCC, ICI monotherapy has shown some tolerable adverse effects such as fatigue, rash, pruritus and increase of serum transaminases that could be managed either by steroid therapy or discontinuation as there were no fatal adverse effects ${ }^{[20,35,52]}$. Several other immune-related adverse events such as pulmonary, gastrointestinal, cardiac, rheumatologic, renal, endocrine, neurologic and dermatologic toxicities have been reported in various cancers treated with $\mathrm{ICI}^{[60]}$. The ideal management of adverse events is to identify these adverse events early with careful monitoring and use of respective treatment options ${ }^{[69]}$. Gastrointestinal toxicities including diarrhoea have been managed with anti-motility agents such as loperamide, diphenoxylate/atropine or higher fibre intake $^{[69]}$. Similarly, the possible liver toxicities post ICI therapy can be managed through liver function tests prior to therapy followed by steroids and mycophenolate mofetil if necessary ${ }^{[70]}$. These toxicities can be rare in incidence but clinicians should monitor these events and act promptly for proper management ${ }^{[69]}$.

Another important limitation of immune checkpoint blockade therapy is poor response to ICI therapy whereby the patient fails to respond after the initial therapy or the patient develops resistance to ICI following initial response ${ }^{[67]}$. In hepato-pancreatic-biliary cancers, a majority of patients fail to respond to ICI therapy ${ }^{[7]]}$. The failure of ICI therapy can result from three factors: (1) mutations of the immunogenicity of cancer itself leading to variable expression of immune related components; (2) redundancy due to expression of other immune checkpoint molecules besides the targeted molecule; and (3) decreased T-cell infiltration ${ }^{[46,72,73]}$. A study by Gopalakrishnan et al. ${ }^{[74]}$ reported that the gut microbiome altered melanoma patient response to anti PD-1 ICIs. Similarly, another study in liver cancer revealed that the gut microbiome utilizes bile acid to regulate immune responses ${ }^{[75]}$.

The expression of immune checkpoint molecules varies among individuals suggesting the need for predictive biomarker to improve the efficacy of ICI therapy ${ }^{[2]}$. The expression of PD-L1 and tumorinfiltrating lymphocytes has been reported to be associated with success of ICI therapy ${ }^{[2]}$. The FDA has approved an IHC test for PD-L1 expression as a predictive biomarker ${ }^{[76]}$. However, some patients with low PD-L1 expression responded well to Nivolumab ${ }^{[7]}$. There is need for more robust predictive biomarkers for ICI therapy besides PD-L1 expression. Tumor mutation burden (TMB), a measure of the overall number of mutations in the tumor specimen, has also been reported as a potential predictive biomarker in ICI therapy ${ }^{[25]}$. Moreover, overexpression of alternative immune checkpoint molecules such as TIM-3 and LAG-3 following anti-PD-1 therapy has been reported ${ }^{[72]}$. In a clinical study of 422 HCC patients, although PD-L1 expression alone lacked predictive power, combining PD-L1 expression with epithelial-to-mesenchymal transition (EMT) phenotype marker expression was associated with poor overall survival and recurrencefree survival ${ }^{[25]}$. As EMT has also been implicated as a resistance mechanism in patients undergoing ICI treatments, a better understanding of this process may aid in overcoming resistance to ICI therapies. Figure 1 summarizes the association between EMT and immune checkpoint regulation and also depicts the EMT process as a main resistance mechanism to immune checkpoint blockade therapy.

\section{ROLE OF EMT IN IMMUNE CHECKPOINT BLOCKADE THERAPY}

EMT is a complex cellular process that enables epithelial cells to gain mesenchymal features resulting in aggressive and motile phenotype ${ }^{[78]}$. The EMT process enables cells to move distances and participate in the formation of internal organs, while the reverse process mesenchymal-to-epithelial transition (MET) enables cells to settle, proliferate and differentiate into different organs once they reach the destination ${ }^{[79-81]}$. EMT is regulated by several factors including transcription factors such as Snail, Twist, zinc-finger E-boxbinding transcription factor, ZEB and other ${ }^{[78,82]}$. EMT is often induced by various cell signalling pathways such as TGF- $\beta$, Wnt, STAT and NOTCH pathways ${ }^{[83]}$. The process of EMT induces epithelial carcinoma cells to transition to metastatic tumor cells such that tumor cells spread from their primary site to a new 


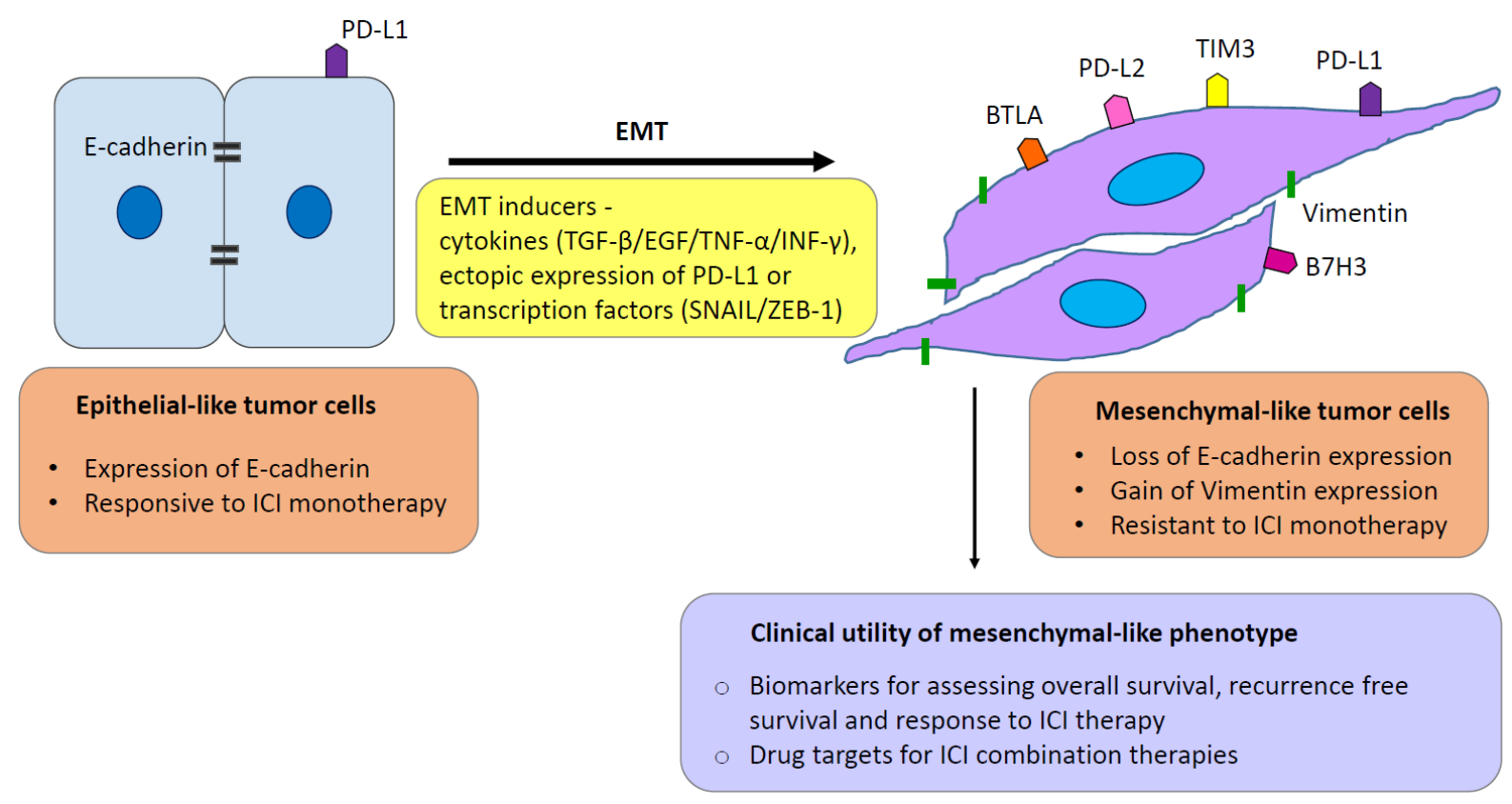

Figure 1. Interconnection between EMT and immune checkpoint based immunotherapy. The diagram illustrates the transition of epithelial-like tumor cells toward a mesenchymal phenotype is associated with immune checkpoint regulation. EMT is induced by several factors including cytokines, upregulation of transcription factors and immune checkpoint molecule PD-L1. EMT is accompanied by the modulation of well-known EMT markers, the loss of epithelial marker E-cadherin and gain of mesenchymal marker Vimentin. Mesenchymal-like tumor cells with elevated expression of different immune checkpoint molecules are more resistant to ICI therapy compared with epithelial-like tumors. The coexistence of features of EMT and expression of immune checkpoint molecules opens the possibility of a mechanistic link between these processes and EMT markers in combination with immune checkpoint molecules can be studied in a prognostic or therapeutic context. PD-L1: programmed death protein ligand -1; EMT: epithelial-to-mesenchymal transition

secondary site where the reverse phenomenon MET enables the metastasized tumor cells to proliferate and differentiate to form secondary tumors ${ }^{[84,85]}$. Accumulating evidence implicates the process of EMT in promoting immune evasion of cancer cells ${ }^{[78,86]}$.

Several in vivo patient and animal model studies have shown that the activation of EMT in HCC promotes tumor progression and metastasis ${ }^{[87]}$. In vitro studies have shown that TGF- $\beta$-induced EMT activates CXCR4/CXCL12 which in turn contributes to HCC tumor progression ${ }^{[88,89]}$. Another study in a mouse model has reported that miR-181, regulated by TGF- $\beta$, is upregulated in HCC and promotes carcinogenesis $^{\left[{ }^{\circ 0]}\right.}$. The association of EMT and HCC has also been reported in several clinical studies. A study of 123 HCC patient samples reported that the majority of clinically aggressive HCC samples had decreased E-cadherin expression, a marker of EMT status ${ }^{[91]}$. In addition, the study also reported that EMT transcription factors Snail and Twist were associated with poor prognosis in HCC with increased invasive and migratory potential ${ }^{[91]}$. Another study reported that HCC patients with mesenchymal tumor phenotype showed earlier recurrence compared to patients with epithelial phenotypes ${ }^{[92]}$. Moreover, the study also showed that patients with epithelial tumor phenotype were more responsive to Sorafenib ${ }^{[92]}$. Collectively, these studies have demonstrated the pivotal role of EMT in HCC progression.

Accumulating evidence shows that cancer cells undergoing EMT can influence the components of the TME and facilitate immune escape by tumors ${ }^{[86,93]}$. The immune components within the TME are comprised of immunosuppressive cells including MDSCs, cancer-associated fibroblasts, tumor-associated macrophages and Treg cells ${ }^{[94]}$. EMT facilitates immune evasion of tumor cells by influencing these immunosuppressive TME cells. For instance, EMT promotes an immunosuppressive TME by recruitment of tumor-associated macrophages through regulation of cytokines ${ }^{[95]}$. EMT also contributes to immunosuppression through 
regulation of immune checkpoint molecules as reported in several instances earlier in this review. EMT is also known to promote immune resistance to NK cell-mediated lysis ${ }^{[94]}$. An EMT inducer, TGF- $\beta$, promotes immunosuppression by several mechanisms including impaired maturation, differentiation or activation of innate and adaptive immune cells, inhibition of cytotoxic T-cell functions and dysregulating cytokine production ${ }^{[94]}$. The association of EMT and immunosuppression in tumor cells has also been reported in HCC. A study reported that hypoxia-induced EMT promotes overexpression of CCL20 resulting in reduced $\mathrm{CD}^{+}$and $\mathrm{CD}_{8}{ }^{+} \mathrm{T}$-cell proliferation along with increased immunosuppressive Treg cells ${ }^{[96]}$. A study has reported that Snail-induced EMT is associated with immunosuppression in cancer patients ${ }^{[86]}$.

In addition, a study has shown that there is an association between EMT score of tumor cells and expression of immune checkpoint molecules such as PD-1, PD-L1, PD-L2, B7-H3 and others ${ }^{[97]}$. Several lung cancer studies have reported the association between EMT and immune checkpoint molecules. One of the earlier studies in lung adenocarcinoma reported that EMT was strongly associated with upregulation of multiple targetable immune checkpoint molecules such as PD-1, PD-L1, PD-2, CTLA-4, BTLA, B7-H3 and TIM-3 ${ }^{[98]}$. Similarly, another study in lung adenocarcinoma demonstrated that EMT phenotype was related to PD-L1 overexpression ${ }^{[99]}$. Notably, a significant correlation between mesenchymal phenotype with expression of immune checkpoint molecules such as PD-1, PD-L1, CTLA-4, OX40L and PD-L2 was confirmed in lung cancer ${ }^{[97]}$. MUC1-C has been reported to simultaneously induce EMT and the expression of PD-L1 in non-small cell lung cancer ${ }^{[93]}$. In lung cancer cell lines, induction of EMT through downregulation of miR-200s and ZEB1 overexpression resulted in increased PD-L1 expression ${ }^{[100]}$. A very interesting study by David et al. ${ }^{[101]}$ utilized M7824, a bifunctional fusion protein, inhibiting PD-L1 and TGF- $\beta$ to demonstrate that TGF- $\beta$-induced immunosuppression in non-small cell lung cancer was mediated by PD-L1 upregulation. Chae et al. ${ }^{[102]}$ reported reduced infiltration of immune cells with antitumor functions and increased infiltration of immune cells with immunosuppressive functions in mesenchymal non-small cell lung cancer. This study further reported increased expression of immune checkpoint molecules CTLA-4 and TIM-3 in mesenchymal lung adenocarcinoma and lung squamous cell carcinoma ${ }^{[102]}$.

Furthermore, PD-L1 expression was closely related with EMT as higher PD-L1 expression was observed in oral squamous cell carcinoma cells co-cultured with mesenchymal phenotypes ${ }^{[103]}$. In breast cancer, Noman et al. ${ }^{[104]}$ revealed that ZEB-1/miR200 or Snail simultaneously induced EMT and upregulated the expression of PD-L1. Chen et al. ${ }^{[105]}$ demonstrated that EMT positive human esophageal cancer tissues had higher PD-L1 expression compared to an EMT negative subgroup. Similar studies have shown an association between EMT and immune checkpoint expression in several cancers including thymic carcinoma $^{[106]}$, melanoma ${ }^{[107]}$, adeno cystic carcinoma ${ }^{[108]}$, extrahepatic cholangiocarcinoma ${ }^{[109]}$ and renal cell carcinoma $^{[110]}$.

Many studies have reported several pathways involved in the regulation of PD-L1 by EMT. PD-L1 expression in non-small cell lung carcinoma was regulated by DNA methylation in a TGF- $\beta 1$ dependent manner and by NF- $\mathrm{KB} / \mathrm{IKK} \varepsilon$ signalling pathway in a TNF- $\alpha$ dependent manner ${ }^{[111]}$. Another study in lung cancer demonstrated that $p$-Smad2 dependent TGF- $\beta$ signalling is involved in PD-L1 overexpression ${ }^{[101]}$. Epidermal growth factor also induced EMT and PD-L1 expression in breast cancer and salivary adenoid cystic carcinoma cells ${ }^{[108,112]}$.

In HCC, a significant association of EMT phenotype with PD-L1 expression was reported in 422 HCC patients $^{[25]}$. The study confirmed that high risk HCC patients had significantly higher expression of mesenchymal marker Vimentin and lower expression of the epithelial marker E-cadherin along with elevated expression of $P D-L 1^{[25]}$. Moreover, the combined coordinate expression of $P D-L 1$ with E-cadherin and Vimentin was associated with poor overall survival and recurrence-free survival ${ }^{[25]}$. This study suggested that patients with an EMT phenotype may benefit from PD-1/PD-L1 blockade therapy. In vitro 
studies demonstrating the direct link between EMT and immune checkpoint expression in HCC are currently lacking.

A few studies have examined the regulation of immune checkpoints in HCC with cytokines that are known to induce EMT, but no EMT markers were evaluated in these studies. One such study in HCC identified that blocking PD-L1 and TGF- $\beta$ enhanced the immune response against tumor suggesting the combination approach of ICIs and TGF- $\beta$ inhibitor drugs ${ }^{[113]}$. The crosstalk between cytokines interferon (IFN)- $\gamma$ and TNF- $\alpha$ was shown to synergistically regulate PD-L1 expression in HCC cells ${ }^{[114]}$. Brown et al.$^{[15]}$ reported that resistance to ICI therapy in HCC was dependent upon overexpression of an immune checkpoint molecule, IDO-1. The authors demonstrated that IDO inhibitors could improve the efficacy and response to ICI therapy ${ }^{[15]}$. Although a few studies have explored the role of EMT in regulating immune checkpoints in HCC, further studies are warranted in this area. A better understanding of how EMT confers resistance to HCC cells treated with ICIs will enable us to develop more effective treatments for HCC.

Immunotherapy, in particular ICI therapy, has revolutionized the treatment approach in several cancers including HCC. ICI treatment is the best alternative in advanced HCC where other curative treatments are not applicable and when systemic therapies fail ${ }^{[20,25]}$. Several ICI clinical trials are underway for HCC, the majority of them target PD-1, PD-L1 and CTLA-4 as monotherapy or in combination with other ICIs or molecular targeted agents ${ }^{[28]}$. Recent studies have identified several novel immune checkpoint molecules that can be potential targets in HCC ${ }^{[25,116,117]}$.

Despite the clinical breakthrough of ICIs in HCC treatment, the response rate is unsatisfactory with a few adverse effects $^{[28]}$. In addition, the resistance to ICI therapy has also limited the use of ICIs in a large patient population ${ }^{[72]}$. The challenge with ICI therapy is to increase the proportion of patients who may gain clinical benefits from this therapy ${ }^{[38]}$. The use of combination therapy with other ICIs may prove to be beneficial as studies report the emergence of alternative checkpoint molecules reduce the response to ICI therapy ${ }^{[72]}$. The efficacy of ICI therapy can be improved by early identification and management of adverse events ${ }^{[118]}$. The selection of patient population who might respond to ICIs is another challenge of ICI therapy ${ }^{[119]}$. Several predictive biomarkers have been utilized such as expression of immune checkpoint molecules (PD-L1 expression by IHC) and $\mathrm{TMB}^{[25,120]}$. However, there are limitations to these biomarkers. Studies have shown that PD-L1 negative patients also respond to anti-PD-1 and anti-PD-L1 treatments ${ }^{[120,121]}$. In addition, it has been reported that patients with a lower number of mutations also benefited from ICI therapy along with patients with higher mutational load ${ }^{[120,122]}$. Thus, there is an urgent need for better predictive biomarkers to improve efficacy of ICI therapy.

Recent studies in several cancers have identified the role of EMT in regulation of immune checkpoint expression. The association between EMT and immune checkpoint expression suggests the utility of EMT status as a potential predictive biomarker in ICI therapy. In addition, EMT inhibitors in combination with ICI may be a potential combination therapy to improve efficacy of ICI therapy in HCC. A few studies have investigated the potential benefits of targeting both EMT and immune checkpoint molecules by utilizing a fusion protein or antibodies targeting TGF- $\beta$ and PD-L $1^{[101,123]}$. Drugs such as Silimarin and Apatinib have been identified that could block both PD-L1 expression and EMT in non-small cell lung cancer and osteoscaroma suggesting similar potential in $\mathrm{HCC}^{[114,124]}$. Collectively, inhibiting the EMT process could increase the sensitivity to ICI treatments and both In vitro and in vivo HCC studies in this area will lay the foundation for future clinical trials.

\section{CONCLUSION}

The emergence of ICIs has provided much hope in improved cancer therapy in several malignancies including HCC. The majority of clinical studies for HCC are based on a few ICIs either as monotherapy or 
combination therapy. There have not been sufficient studies exploring novel immune checkpoint molecules and predictive biomarkers for ICIs in HCC. The relationship between EMT and immune checkpoint molecules presents a promising combinatorial approach for the treatment of HCC.

\section{DECLARATIONS}

\section{Authors' contributions}

Made substantial contributions to conception and design of the study, manuscript writing: Shrestha R, Jayachandran A

Manuscript revision and interpretation: Shrestha R, Bridle KR, Crawford D, Jayachandran A

Final approval of manuscripts: Shrestha R, Bridle KR, Crawford D, Jayachandran A

\section{Availability of data and materials}

Not applicable.

\section{Financial support and sponsorship}

This manuscript publication is funded by Gallipoli Medical Research Foundation.

\section{Conflicts of interest}

All authors declared that there are no conflicts of interest.

\section{Ethical approval and consent to participate}

Not applicable.

\section{Consent for publication}

Not applicable.

\section{Copyright}

(c) The Author(s) 2019.

\section{REFERENCES}

1. Chacko S, Samanta S. "Hepatocellular carcinoma: A life-threatening diseasen". Biomed Pharmacother 2016;84:1679-88.

2. Galun D, Srdic-Rajic T, Bogdanovic A, Loncar Z, Zuvela M. Targeted therapy and personalized medicine in hepatocellular carcinoma: drug resistance, mechanisms, and treatment strategies. J Hepatocell Carcinoma 2017;4:93-103.

3. Tinkle CL, Haas-Kogan D. Hepatocellular carcinoma: natural history, current management, and emerging tools. Biologics 2012;6:207-19.

4. Tian M, Shi Y, Liu W, Fan J. Immunotherapy of hepatocellular carcinoma: strategies for combinatorial intervention. Sci China Life Sci 2019; DOI: 10.1007/s11427-018-9446-2.

5. Ueno M, Hayami S, Shigekawa Y, Kawai M, Hirono S, et al. Prognostic impact of surgery and radiofrequency ablation on single nodular HCC $\leq 5 \mathrm{~cm}$ : Cohort study based on serum HCC markers. J Hepatol 2015;63:1352-9.

6. Buonaguro L, Mauriello A, Cavalluzzo B, Petrizzo A, Tagliamonte M. Immunotherapy in hepatocellular carcinoma. Ann Hepatol 2019;18:291-7.

7. Crocetti L, Bargellini I, Cioni R. Loco-regional treatment of HCC: current status. Clin Radiol 2017;72:626-35.

8. Llovet JM, Real MI, Montaña X, Planas R, Coll S, et al; Group BLC. Arterial embolisation or chemoembolisation versus symptomatic treatment in patients with unresectable hepatocellular carcinoma: a randomised controlled trial. Lancet 2002;359:1734-9.

9. Kudo M, Finn RS, Qin S, Han KH, Ikeda K, et al. Lenvatinib versus sorafenib in first-line treatment of patients with unresectable hepatocellular carcinoma: a randomised phase 3 non-inferiority trial. Lancet 2018;391:1163-73.

10. Mir N, Jayachandran A, Dhungel B, Shrestha R, Steel JC. Epithelial-to-Mesenchymal Transition: A Mediator of Sorafenib Resistance in Advanced Hepatocellular Carcinoma. Curr Cancer Drug Targets 2017;17:698-706.

11. Kudo M. Targeted and immune therapies for hepatocellular carcinoma: Predictions for 2019 and beyond. World J Gastroenterol 2019;25:789-807.

12. Abou-Alfa GK, Meyer T, Cheng AL, El-Khoueiry AB, Rimassa L, et al. Cabozantinib in Patients with Advanced and Progressing Hepatocellular Carcinoma. N Engl J Med 2018;379:54-63.

13. Bruix J, Qin S, Merle P, Granito A, Huang YH, et al. Regorafenib for patients with hepatocellular carcinoma who progressed on 
sorafenib treatment (RESORCE): a randomised, double-blind, placebo-controlled, phase 3 trial. Lancet 2017;389:56-66.

14. Kudo M. Systemic Therapy for Hepatocellular Carcinoma: Latest Advances. Cancers 2018;10:412.

15. Zhu AX, Kang Y-K, Yen C-J, Finn RS, Galle PR, et al. REACH-2: A randomized, double-blind, placebo-controlled phase 3 study of ramucirumab versus placebo as second-line treatment in patients with advanced hepatocellular carcinoma (HCC) and elevated baseline alpha-fetoprotein (AFP) following first-line sorafenib. J Clin Oncol 2018;36:4003.

16. Floudas CS, Brar G, Greten TF. Immunotherapy: Current Status and Future Perspectives. Dig Dis Sci 2019;64:1030-40.

17. Herbst RS, Baas P, Kim DW, Felip E, Pérez-Gracia JL, et al. Pembrolizumab versus docetaxel for previously treated, PD-L1-positive, advanced non-small-cell lung cancer (KEYNOTE-010): a randomised controlled trial. Lancet 2016;387:1540-50.

18. Hodi FS, O'Day SJ, McDermott DF, Weber RW, Sosman JA, et al. Improved survival with ipilimumab in patients with metastatic melanoma. N Engl J Med 2010;363:711-23.

19. Topalian SL, Drake CG, Pardoll DM. Immune checkpoint blockade: a common denominator approach to cancer therapy. Cancer Cell 2015;27:450-61.

20. El-Khoueiry AB, Sangro B, Yau T, Crocenzi TS, Kudo M, et al. Nivolumab in patients with advanced hepatocellular carcinoma (CheckMate 040): an open-label, non-comparative, phase 1/2 dose escalation and expansion trial. Lancet 2017;389:2492-502.

21. Siu EH, Chan AW, Chong CC, Chan SL, Lo KW, et al. Treatment of advanced hepatocellular carcinoma: immunotherapy from checkpoint blockade to potential of cellular treatment. Transl Gastroenterol Hepatol 2018;3:89.

22. Zhu AX, Finn RS, Edeline J, Cattan S, Ogasawara S, et al. Pembrolizumab in patients with advanced hepatocellular carcinoma previously treated with sorafenib (KEYNOTE-224): a non-randomised, open-label phase 2 trial. Lancet Oncol 2018;19:940-52.

23. Hato T, Goyal L, Greten TF, Duda DG, Zhu AX. Immune checkpoint blockade in hepatocellular carcinoma: current progress and future directions. Hepatology 2014;60:1776-82.

24. Pardoll DM. The blockade of immune checkpoints in cancer immunotherapy. Nat Rev Cancer 2012;12:252-64.

25. Shrestha R, Prithviraj P, Anaka M, Bridle KR, Crawford DHG, et al. Monitoring Immune Checkpoint Regulators as Predictive Biomarkers in Hepatocellular Carcinoma. Front Oncol 2018;8:269.

26. Webb ES, Liu P, Baleeiro R, Lemoine NR, Yuan M, et al. Immune checkpoint inhibitors in cancer therapy. J Biomed Res 2018;32:317-26.

27. Ardolino L, Joshua A. Immune checkpoint inhibitors in malignancy. Aust Prescr 2019;42:62-7.

28. Okusaka T, Ikeda M. Immunotherapy for hepatocellular carcinoma: current status and future perspectives. ESMO Open 2018;3:e00455.

29. Pardee AD, Butterfield LH. Immunotherapy of hepatocellular carcinoma: Unique challenges and clinical opportunities. Oncoimmunology 2012;1:48-55.

30. Kapanadze T, Gamrekelashvili J, Ma C, Chan C, Zhao F, et al. Regulation of accumulation and function of myeloid derived suppressor cells in different murine models of hepatocellular carcinoma. J Hepatol 2013;59:1007-13.

31. Jenne CN, Kubes P. Immune surveillance by the liver. Nat Immunol 2013;14:996-1006.

32. Okrah K, Tarighat S, Liu B, Koeppen H, Wagle MC, et al. Transcriptomic analysis of hepatocellular carcinoma reveals molecular features of disease progression and tumor immune biology. NPJ Precis Oncol 2018;2:25.

33. Schneider H, Downey J, Smith A, Zinselmeyer BH, Rush C, et al. Reversal of the TCR stop signal by CTLA-4. Science 2006;313:1972-5.

34. Mizukoshi E, Nakamoto Y, Arai K, Yamashita T, Sakai A, et al. Comparative analysis of various tumor-associated antigen-specific t-cell responses in patients with hepatocellular carcinoma. Hepatology 2011;53:1206-16.

35. Sangro B, Gomez-Martin C, de la Mata M, Iñarrairaegui M, Garralda E, et al. A clinical trial of CTLA-4 blockade with tremelimumab in patients with hepatocellular carcinoma and chronic hepatitis C. J Hepatol 2013;59:81-8.

36. Brar G, Greten TF, Brown ZJ. Current frontline approaches in the management of hepatocellular carcinoma: the evolving role of immunotherapy. Therap Adv Gastroenterol 2018;11:1756284818808086.

37. Iñarrairaegui M, Melero I, Sangro B. Immunotherapy of Hepatocellular Carcinoma: Facts and Hopes. Clin Cancer Res 2018;24:1518-24.

38. Liu X, Qin S. Immune Checkpoint Inhibitors in Hepatocellular Carcinoma: Opportunities and Challenges. Oncologist 2019;24:S3-S10.

39. Finn RS, Ryoo B-Y, Merle P, Kudo M, Bouattour M, et al. Results of KEYNOTE-240: phase 3 study of pembrolizumab (Pembro) vs best supportive care (BSC) for second line therapy in advanced hepatocellular carcinoma (HCC). J Clin Oncol 2019;37:4004.

40. Busato D, Mossenta M, Baboci L, Di Cintio F, Toffoli G, et al. Novel immunotherapeutic approaches for hepatocellular carcinoma treatment. Expert Rev Clin Pharmacol 2019;12:453-70.

41. Qin S, Finn RS, Kudo M, Meyer T, Vogel A, et al. A phase 3, randomized, open-label, multicenter study to compare the efficacy and safety of tislelizumab, an anti-PD-1 antibody, versus sorafenib as first-line treatment in patients with advanced hepatocellular carcinoma. J Clin Oncol 2018;36:TPS3110.

42. Huang J, Mo H, Wu D, Chen X, Ma L, et al. Phase I study of the anti-PD-1 antibody SHR-1210 in patients with advanced solid tumors. J Clin Oncol 2017;35:e15572.

43. Xu J, Zhang Y, Jia R, Yue C, Chang L, et al. Anti-PD-1 Antibody SHR-1210 Combined with Apatinib for Advanced Hepatocellular Carcinoma, Gastric, or Esophagogastric Junction Cancer: An Open-label, Dose Escalation and Expansion Study. Clin Cancer Res 2019;25:515-23.

44. Qin SK, Ren ZG, Meng ZQ, Chen ZD, Chai XL, et al. LBA27A randomized multicentered phase II study to evaluate SHR-1210 (PD-1 antibody) in subjects with advanced hepatocellular carcinoma (HCC) who failed or intolerable to prior systemic treatment. Ann Oncol 2018;29:mdy424.029.

45. Butte MJ, Keir ME, Phamduy TB, Sharpe AH, Freeman GJ. Programmed death-1 ligand 1 interacts specifically with the B7-1 costimulatory molecule to inhibit T cell responses. Immunity 2007;27:111-22. 
46. Xu F, Jin T, Zhu Y, Dai C. Immune checkpoint therapy in liver cancer. J Exp Clin Cancer Res 2018;37:110.

47. Calderaro J, Rousseau B, Amaddeo G, Mercey M, Charpy C, et al. Programmed death ligand 1 expression in hepatocellular carcinoma: Relationship With clinical and pathological features. Hepatology 2016;64:2038-46.

48. Chang H, Jung W, Kim A, Kim HK, Kim WB, et al. Expression and prognostic significance of programmed death protein 1 and programmed death ligand-1, and cytotoxic T lymphocyte-associated molecule-4 in hepatocellular carcinoma. APMIS 2017;125:690-8.

49. Sideras K, Biermann K, Verheij J, Takkenberg BR, Mancham S, et al. PD-L1, Galectin-9 and CD8. Oncoimmunology 2017;6:e1273309.

50. Wu K, Kryczek I, Chen L, Zou W, Welling TH. Kupffer cell suppression of CD8+ T cells in human hepatocellular carcinoma is mediated by B7-H1/programmed death-1 interactions. Cancer Res 2009;69:8067-75.

51. Wainberg ZA, Segal NH, Jaeger D, Lee K-H, Marshall J, et al. Safety and clinical activity of durvalumab monotherapy in patients with hepatocellular carcinoma (HCC). J Clin Oncol 2017;35:4071.

52. Nishida N, Kudo M. Immune checkpoint blockade for the treatment of human hepatocellular carcinoma. Hepatol Res 2018;48:622-34.

53. Cella D, Grünwald V, Escudier B, Hammers HJ, George S, et al. Patient-reported outcomes of patients with advanced renal cell carcinoma treated with nivolumab plus ipilimumab versus sunitinib (CheckMate 214): a randomised, phase 3 trial. Lancet Oncol 2019;20:297-310.

54. Jain S, Clark JI. Ipilimumab for the treatment of melanoma. Melanoma Manag 2015;2:33-9.

55. Kelley RK, Abou-Alfa GK, Bendell JC, Kim T-Y, Borad MJ, et al. Phase I/II study of durvalumab and tremelimumab in patients with unresectable hepatocellular carcinoma (HCC): Phase I safety and efficacy analyses. J Clin Oncol 2017;35:4073.

56. Abou-Alfa GK, Chan SL, Furuse J, Galle PR, Kelley RK, et al. A randomized, multicenter phase 3 study of durvalumab (D) and tremelimumab $(\mathrm{T})$ as first-line treatment in patients with unresectable hepatocellular carcinoma (HCC): HIMALAYA study. J Clin Oncol 2018;36:TPS4144.

57. Finn RS, Ducreux M, Qin S, Galle PR, Zhu AX, et al. IMbrave150: A randomized phase III study of 1L atezolizumab plus bevacizumab vs sorafenib in locally advanced or metastatic hepatocellular carcinoma. J Clin Oncol 2018;36:TPS4141.

58. J Pishvaian M, S Lee M, Ryoo BY, Stein S, Lee KH, et al. LBA26Updated safety and clinical activity results from a phase Ib study of atezolizumab + bevacizumab in hepatocellular carcinoma (HCC); Ann Oncol 2018;29; DOI:10.1093/annonc/mdy424.028.

59. Ikeda M, Sung MW, Kudo M, Kobayashi M, Baron AD, et al. A phase $1 \mathrm{~b}$ trial of lenvatinib (LEN) plus pembrolizumab (PEM) in patients (pts) with unresectable hepatocellular carcinoma (uHCC). J Clin Oncol 2018;36:4076.

60. Xue JM, Astère M, Zhong MX, Lin H, Shen J, et al. Efficacy and safety of apatinib treatment for gastric cancer, hepatocellular carcinoma and non-small cell lung cancer: a meta-analysis. Onco Targets Ther 2018;11:6119-28.

61. Deng L, Liang H, Burnette B, Beckett M, Darga T, et al. Irradiation and anti-PD-L1 treatment synergistically promote antitumor immunity in mice. J Clin Invest 2014;124:687-95.

62. Kreidieh M, Zeidan YH, Shamseddine A. The Combination of Stereotactic Body Radiation Therapy and Immunotherapy in Primary Liver Tumors. J Oncol 2019;2019:4304817.

63. Harding JJ, Erinjeri JP, Tan BR, Reiss KA, Mody K, et al. A multicenter pilot study of nivolumab (NIVO) with drug eluting bead transarterial chemoembolization (deb-TACE) in patients (pts) with liver limited hepatocellular carcinoma (HCC). J Clin Oncol 2018;36:TPS4146.

64. Duffy AG, Ulahannan SV, Makorova-Rusher O, Rahma O, Wedemeyer H, et al. Tremelimumab in combination with ablation in patients with advanced hepatocellular carcinoma. J Hepatol 2017;66:545-51.

65. Chang EWY, Tai DW-M, Koo S-L, Ng MC, Yeong JPS, et al. A phase II open-label, single-centre, non-randomized trial of Y90 transarterial radioembolization in combination with nivolumab in Asian patients with intermediate stage hepatocellular carcinoma: An immunological study of radioembolization in combination with anti-PD1 therapy in HCC. J Clin Oncol 2018;36:TPS542.

66. Hecht JR, Prat A, Pless M, Cubillo A, Calvo A, et al. A phase 1b/2, multicenter, open-label trial to evaluate the safety of talimogene laherparepvec (T-VEC) injected into primary and metastatic liver tumors alone and in combination with pembrolizumab (pembro) (MASTERKEY-318). J Clin Oncol 2018;36:TPS3105.

67. Jenkins RW, Barbie DA, Flaherty KT. Mechanisms of resistance to immune checkpoint inhibitors. Br J Cancer 2018;118:9-16.

68. Johnson DB, Balko JM, Compton ML, Chalkias S, Gorham J, et al. Fulminant Myocarditis with Combination Immune Checkpoint Blockade. N Engl J Med 2016;375:1749-55.

69. Winer A, Bodor JN, Borghaei H. Identifying and managing the adverse effects of immune checkpoint blockade. J Thorac Dis 2018;10:S480-S9.

70. Puzanov I, Diab A, Abdallah K, Bingham CO, Brogdon C, et al; Group SfIoCTMW. Managing toxicities associated with immune checkpoint inhibitors: consensus recommendations from the Society for Immunotherapy of Cancer (SITC) Toxicity Management Working Group. J Immunother Cancer 2017;5:95.

71. McLoughlin KC, Brown ZJ, Shukla Y, Shukla V. Promise and pitfalls of immune checkpoint inhibitors in hepato-pancreato-biliary malignancies. Discov Med 2018;26:85-92.

72. Koyama S, Akbay EA, Li YY, Herter-Sprie GS, Buczkowski KA, et al. Adaptive resistance to therapeutic PD-1 blockade is associated with upregulation of alternative immune checkpoints. Nat Commun 2016;7:10501.

73. Peng W, Chen JQ, Liu C, Malu S, Creasy C, et al. Loss of PTEN Promotes Resistance to T Cell-Mediated Immunotherapy. Cancer Discov 2016;6:202-16.

74. Gopalakrishnan V, Spencer CN, Nezi L, Reuben A, Andrews MC, et al. Gut microbiome modulates response to anti-PD-1 immunotherapy in melanoma patients. Science 2018;359:97-103.

75. Ma C, Han M, Heinrich B, Fu Q, Zhang Q, et al. Gut microbiome-mediated bile acid metabolism regulates liver cancer via NKT cells. 
Science 2018;360:858

76. Sul J, Blumenthal GM, Jiang X, He K, Keegan P, et al. FDA Approval Summary: Pembrolizumab for the Treatment of Patients With Metastatic Non-Small Cell Lung Cancer Whose Tumors Express Programmed Death-Ligand 1. Oncologist 2016;21:643-50.

77. Ma K, Jin Q, Wang M, Li X, Zhang Y. Research progress and clinical application of predictive biomarker for immune checkpoint inhibitors. Expert Rev Mol Diagn 2019;19:517-29.

78. Kalluri R, Weinberg RA. The basics of epithelial-mesenchymal transition. J Clin Invest 2009;119:1420-8.

79. Acloque H, Adams MS, Fishwick K, Bronner-Fraser M, Nieto MA. Epithelial-mesenchymal transitions: the importance of changing cell state in development and disease. J Clin Invest 2009;119:1438-49.

80. Rao SR, Jayachandran A. Epithelial-to-mesenchymal transition as a potential target for antineoplastic therapies. J Cancer Clin Trials 2016;1:e103.

81. Thiery JP, Acloque H, Huang RY, Nieto MA. Epithelial-mesenchymal transitions in development and disease. Cell 2009;139:871-90.

82. Jayachandran A, Shrestha R, Dhungel B, Huang IT, Vasconcelos MYK, et al. Murine hepatocellular carcinoma derived stem cells reveal epithelial-to-mesenchymal plasticity. World J Stem Cells 2017;9:159-68.

83. Gonzalez DM, Medici D. Signaling mechanisms of the epithelial-mesenchymal transition. Sci Signal 2014;7:re8.

84. Jayachandran A, Dhungel B, Steel JC. Epithelial-to-mesenchymal plasticity of cancer stem cells: therapeutic targets in hepatocellular carcinoma. J Hematol Oncol 2016;9:74.

85. Singh M, Yelle N, Venugopal C, Singh SK. EMT: Mechanisms and therapeutic implications. Pharmacol Ther 2018;182:80-94.

86. Kudo-Saito C, Shirako H, Takeuchi T, Kawakami Y. Cancer metastasis is accelerated through immunosuppression during Snailinduced EMT of cancer cells. Cancer Cell 2009;15:195-206.

87. Giannelli G, Koudelkova P, Dituri F, Mikulits W. Role of epithelial to mesenchymal transition in hepatocellular carcinoma. J Hepatol 2016;65:798-808.

88. Bertran E, Caja L, Navarro E, Sancho P, Mainez J, et al. Role of CXCR4/SDF-1 alpha in the migratory phenotype of hepatoma cells that have undergone epithelial-mesenchymal transition in response to the transforming growth factor-beta. Cell Signal 2009;21:1595606.

89. Bertran E, Crosas-Molist E, Sancho P, Caja L, Lopez-Luque J, et al. Overactivation of the TGF- $\beta$ pathway confers a mesenchymal-like phenotype and CXCR4-dependent migratory properties to liver tumor cells. Hepatology 2013;58:2032-44.

90. Wang B, Hsu SH, Majumder S, Kutay H, Huang W, et al. TGFbeta-mediated upregulation of hepatic miR-181b promotes hepatocarcinogenesis by targeting TIMP3. Oncogene 2010;29:1787-97.

91. Wang MH, Sun R, Zhou XM, Zhang MY, Lu JB, et al. Epithelial cell adhesion molecule overexpression regulates epithelialmesenchymal transition, stemness and metastasis of nasopharyngeal carcinoma cells via the PTEN/AKT/mTOR pathway. Cell Death Dis 2018;9:2.

92. Yamada S, Okumura N, Wei L, Fuchs BC, Fujii T, et al. Epithelial to mesenchymal transition is associated with shorter disease-free survival in hepatocellular carcinoma. Ann Surg Oncol 2014;21:3882-90.

93. Bouillez A, Rajabi H, Jin C, Samur M, Tagde A, et al. MUC1-C integrates PD-L1 induction with repression of immune effectors in non-small-cell lung cancer. Oncogene 2017;36:4037-46.

94. Terry S, Savagner P, Ortiz-Cuaran S, Mahjoubi L, Saintigny P, et al. New insights into the role of EMT in tumor immune escape. Mol Oncol 2017;11:824-46.

95. Soundararajan R, Fradette JJ, Konen JM, Moulder S, Zhang X, et al. Targeting the Interplay between Epithelial-to-MesenchymalTransition and the Immune System for Effective Immunotherapy. Cancers 2019;11:714.

96. Ye LY, Chen W, Bai XL, Xu XY, Zhang Q, et al. Hypoxia-Induced Epithelial-to-Mesenchymal Transition in Hepatocellular Carcinoma Induces an Immunosuppressive Tumor Microenvironment to Promote Metastasis. Cancer Res 2016;76:818-30.

97. Mak MP, Tong P, Diao L, Cardnell RJ, Gibbons DL, et al. A Patient-Derived, Pan-Cancer EMT Signature Identifies Global Molecular Alterations and Immune Target Enrichment Following Epithelial-to-Mesenchymal Transition. Clin Cancer Res 2016;22:609-20.

98. Lou Y, Diao L, Cuentas ER, Denning WL, Chen L, et al. Epithelial-Mesenchymal Transition Is Associated with a Distinct Tumor Microenvironment Including Elevation of Inflammatory Signals and Multiple Immune Checkpoints in Lung Adenocarcinoma. Clin Cancer Res 2016;22:3630-42.

99. Kim S, Koh J, Kim MY, Kwon D, Go H, et al. PD-L1 expression is associated with epithelial-to-mesenchymal transition in adenocarcinoma of the lung. Hum Pathol 2016;58:7-14.

100. Chen L, Gibbons DL, Goswami S, Cortez MA, Ahn YH, et al. Metastasis is regulated via microRNA-200/ZEB1 axis control of tumour cell PD-L1 expression and intratumoral immunosuppression. Nat Commun 2014;5:5241.

101. David JM, Dominguez C, McCampbell KK, Gulley JL, Schlom J, et al. A novel bifunctional anti-PD-L1/TGF- $\beta$ Trap fusion protein (M7824) efficiently reverts mesenchymalization of human lung cancer cells. Oncoimmunology 2017;6:e1349589.

102. Chae YK, Chang S, Ko T, Anker J, Agte S, et al. Epithelial-mesenchymal transition (EMT) signature is inversely associated with T-cell infiltration in non-small cell lung cancer (NSCLC). Sci Rep 2018;8:2918.

103. Hirai M, Kitahara H, Kobayashi Y, Kato K, Bou-Gharios G, et al. Regulation of PD-L1 expression in a high-grade invasive human oral squamous cell carcinoma microenvironment. Int J Oncol 2017;50:41-8.

104. Noman MZ, Janji B, Abdou A, Hasmim M, Terry S, et al. The immune checkpoint ligand PD-L1 is upregulated in EMT-activated human breast cancer cells by a mechanism involving ZEB-1 and miR-200. Oncoimmunology 2017;6:e1263412.

105. Chen L, Xiong Y, Li J, Zheng X, Zhou Q, et al. PD-L1 Expression Promotes Epithelial to Mesenchymal Transition in Human Esophageal Cancer. Cell Physiol Biochem 2017;42:2267-80. 
106. Funaki S, Shintani Y, Kawamura T, Kanzaki R, Minami M, et al. Chemotherapy enhances programmed cell death 1/ligand 1 expression via TGF- $\beta$ induced epithelial mesenchymal transition in non-small cell lung cancer. Oncol Rep 2017;38:2277-84.

107. Wicklein D, Otto B, Suling A, Elies E, Lüers G, et al. CEACAM1 promotes melanoma metastasis and is involved in the regulation of the EMT associated gene network in melanoma cells. Sci Rep 2018;8:11893.

108. Wang Y, Hu J, Ye W, Zhang X, Ju H, et al. EGFR activation induced Snail-dependent EMT and myc-dependent PD-L1 in human salivary adenoid cystic carcinoma cells. Cell Cycle 2018;17:1457-70.

109. Ueno T, Tsuchikawa T, Hatanaka KC, Hatanaka Y, Mitsuhashi T, et al. Prognostic impact of programmed cell death ligand 1 (PD-L1) expression and its association with epithelial-mesenchymal transition in extrahepatic cholangiocarcinoma. Oncotarget 2018;9:20034-47.

110. Liang J, Liu Z, Zou Z, Tang Y, Zhou C, et al. The Correlation Between the Immune and Epithelial-Mesenchymal Transition Signatures Suggests Potential Therapeutic Targets and Prognosis Prediction Approaches in Kidney Cancer. Sci Rep 2018;8:6570.

111. Asgarova A, Asgarov K, Godet Y, Peixoto P, Nadaradjane A, et al. PD-L1 expression is regulated by both DNA methylation and NFkB during EMT signaling in non-small cell lung carcinoma. Oncoimmunology 2018;7:e1423170.

112. Li CW, Lim SO, Xia W, Lee HH, Chan LC, et al. Glycosylation and stabilization of programmed death ligand-1 suppresses T-cell activity. Nat Commun 2016;7:12632.

113. Ihling C, Naughton B, Zhang Y, Rolfe PA, Frick-Krieger E, et al. Observational Study of PD-L1, TGF- $\beta$, and Immune Cell Infiltrates in Hepatocellular Carcinoma. Front Med 2019;6:15.

114. Liakopoulou C, Kazazis C, Vallianou NG. Silimarin and Cancer. Anticancer Agents Med Chem 2018;18:1970-4.

115. Brown ZJ, Yu SJ, Heinrich B, Ma C, Fu Q, et al. Indoleamine 2,3-dioxygenase provides adaptive resistance to immune checkpoint inhibitors in hepatocellular carcinoma. Cancer Immunol Immunother 2018;67:1305-15.

116. Shali S, Yu J, Zhang X, Wang X, Jin Y, et al. Ecto-5'-nucleotidase (CD73) is a potential target of hepatocellular carcinoma. J Cell Physiol 2019;234:10248-59.

117. Yuan L, Dong L, Yu G, Fan W, Zhang L, et al. Aberrant expression of B7-H4 may contribute to the development of hepatocellular carcinoma. Mol Med Rep 2016;14:5015-24.

118. Teply BA, Lipson EJ. Identification and management of toxicities from immune checkpoint-blocking drugs. Oncology (Williston Park) 2014;28 Suppl 3:30-8.

119. Signorelli D, Giannatempo P, Grazia G, Aiello MM, Bertolini F, et al. Patients Selection for Immunotherapy in Solid Tumors: Overcome the Naïve Vision of a Single Biomarker. Biomed Res Int 2019;2019:9056417.

120. Sharma P. Immune Checkpoint Therapy and the Search for Predictive Biomarkers. Cancer J 2016;22:68-72.

121. Grosso J, Horak CE, Inzunza D, Cardona DM, Simon JS, et al. Association of tumor PD-L1 expression and immune biomarkers with clinical activity in patients (pts) with advanced solid tumors treated with nivolumab (anti-PD-1; BMS-936558; ONO-4538). J Clin Oncol 2013;31:3016.

122. Rizvi NA, Hellmann MD, Snyder A, Kvistborg P, Makarov V, et al. Mutational landscape determines sensitivity to PD-1 blockade in non-small cell lung cancer. Science 2015;348:124-8.

123. Dodagatta-Marri E, Meyer DS, Reeves MQ, Paniagua R, To MD, et al. $\alpha$-PD-1 therapy elevates Treg/Th balance and increases tumor cell pSmad 3 that are both targeted by $\alpha$-TGF $\beta$ antibody to promote durable rejection and immunity in squamous cell carcinomas. J Immunother Cancer 2019;7:62.

124. Zheng B, Ren T, Huang Y, Guo W. Apatinib inhibits migration and invasion as well as PD-L1 expression in osteosarcoma by targeting STAT3. Biochem Biophys Res Commun 2018;495:1695-701. 